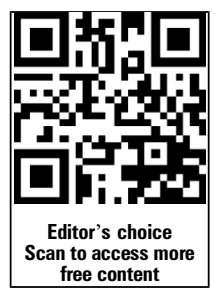

- Additional material is published online only. To view, please visit the journal online (http://dx.doi.org/10.1136/ heartjnl-2015-308848).

${ }^{1}$ Departments of Cardiac Surgery and Cardiology, Royal Children's Hospital, Murdoch Children's Research Institute, University of Melbourne, Melbourne, Australia ${ }^{2}$ Department of Cardiothoracic Surgery, The Alfred Hospital, Monash University, Melbourne, Australia

${ }^{3}$ Department of Cardiology, St Vincent's Hospital, Sydney, Australia

${ }^{4}$ Greenlane Cardiothoracic Unit, Auckland City Hospital, Auckland, New Zealand

\section{Correspondence to}

Professor Igor E Konstantinov, Cardiac Surgery Unit, Royal Children's Hospital, Parkville, VIC 3052, Australia; igor. konstantinov@rch.org.au

Received 16 October 2015 Revised 13 February 2016 Accepted 16 February 2016 Published Online First 21 March 2016

\title{
Heart transplantation in Fontan patients across Australia and New Zealand
}

\author{
William Y Shi, ${ }^{1}$ Matthew S Yong, ${ }^{2}$ David C McGiffin, ${ }^{2}$ Pankaj Jain, ${ }^{3}$ Peter N Ruygrok, ${ }^{4}$ \\ Silvana F Marasco, ${ }^{2}$ Kirsten Finucane, ${ }^{4}$ Anne Keogh, ${ }^{3}$ Yves d'Udekem, ${ }^{1}$ \\ Robert G Weintraub, ${ }^{1}$ Igor E Konstantinov'
}

ABSTRACT

Objective Patients with Fontan physiology may eventually require heart transplantation (HT). We determined the rates and outcomes of HT in a national, population-based multicentre study.

Methods From 1990 to 2015, 1369 patients underwent the Fontan procedure as recorded in the Australia and New Zealand Fontan Registry. We identified those who underwent HT and analysed their outcomes. We compared rates of HT between two catchment areas. In area $1(n=721)$, patients were referred to the national paediatric HT programme or its associated adult programme. In area $2(n=648)$, patients were referred to the national paediatric HT programme or one of the other adult HT programmes.

Results Mean follow-up time post-Fontan was 11 \pm 8 years. Freedom from Fontan failure was $74 \% \pm 3.9 \%$ at 20 years. HT was performed in 34 patients. Patients living in area 1 were more likely to have HT $(4.0 \%, 29 /$ 721 vs $0.8 \%, 5 / 648, p<0.001)$ with a cumulative proportion of $3.4 \%$ vs $0.7 \%$ at 10 years and $6.8 \%$ vs $1.2 \%$ at 20 years $(p=0.002)$. Area 1 patients were more likely to undergo HT (hazard ratio $4.7,95 \% \mathrm{Cl} 1.7$ to $13.5, p=0.003)$ on multivariable regression. Post-HT survival at 1, 5 and 10 years was $91 \%, 78 \%$ and $71 \%$, respectively. Compared with other patients with congenital heart disease $(n=87)$, Fontan patients had similar in-hospital outcomes and long-term survival. Conclusions Although HT after the Fontan procedure can be achieved with excellent outcomes, most patients with Fontan failure do not undergo HT. Significant regional differences in rates of HT in Fontan patients exist.

\section{INTRODUCTION}

Excellent long-term outcomes can be expected after the Fontan completion. ${ }^{1}$ Despite this, a proportion of patients will experience primary myocardial dysfunction or intolerance of the Fontan circulation, and in the absence of a correctable lesion, will require heart transplantation (HT). ${ }^{2}$

Such patients may experience Fontan-specific co-morbidities such as protein-losing enteropathy (PLE), plastic bronchitis and intractable arrhythmias, thrombosis, among others. In addition, the challenges associated with reoperative cardiac surgery and potential requirement for reconstruction of the great arteries may further complicate their management. Such patients are often perceived to be of higher operative risk compared with other potential transplant candidates with congenital heart disease (CHD). ${ }^{3-5}$

The Australia and New Zealand Fontan Registry, the largest of its kind, provides us with a unique opportunity to evaluate management of patients with a failing Fontan circulation. ${ }^{6}$ We determined the rates of transplantation in patients with a Fontan circulation and examined their early and late outcomes. To our knowledge, this study is the first to examine at a national level, the rates of transplantation for patients with a Fontan circulation.

\section{METHODS}

The Australian and New Zealand Fontan Registry was commenced in 2008, and includes patients who underwent the Fontan procedure in both countries. All eight paediatric and seven adult centres overseeing the care of patients with $\mathrm{CHD}$ in Australia and New Zealand participated in this study. The clinical characteristics, early and late outcomes of the patients have been reported previously. ${ }^{1}{ }^{6-8}$ Patients who died prior to discharge after the Fontan procedure, those who underwent Fontan takedown in the same admission and patients referred from overseas were excluded from this study, resulting in 1369 patients for analysis.

The Australia and New Zealand Cardiothoracic Organ Transplant Registry (ANZCOTR) records the details of all heart transplants performed in Australia and New Zealand $(n=2479)$ since 1984. This registry was interrogated for details of all paediatric and adult patients with CHD who received HT.

Using both registries, we identified patients with a Fontan circulation who underwent HT. In addition to information from the two registries, supplementary data were collected from each transplant centre via site visits and entered into a standardised database. Information collected were patient demographics, clinical, intraoperative, postoperative and follow-up details. Only the data from the centres that performed HT for patients with a Fontan circulation were included for comparative analyses. Ethics approval was granted nationally in Australia and New Zealand and by the Research Ethics Committee of participating institutions.

Within Australia and New Zealand, there are six transplant centres. In Australia, the national paediatric HT programme is located at the Royal Children's Hospital (RCH), Melbourne. Local
To cite: Shi WY, Yong MS , et al. Heart

1120 
patients from the $\mathrm{RCH}$ with heart failure have their care transitioned to an adult HT programme based at the Alfred Hospital, Melbourne. In the other Australian states, patients with heart failure are transitioned and followed-up by one of three other adult heart failure programmes in their respective states. In New Zealand, the national paediatric and adult HT services are based at The Starship Children's Hospital and Auckland City Hospital in Auckland, which integrate adult and paediatric programmes at a single site.

To examine referral patterns, we categorised patients into two catchment areas. In area 1, patients are referred to either a national paediatric HT programme or the associated adult HT programme of the same city. In area 2, patients are referred to either a national paediatric HT programme at $\mathrm{RCH}$ or one of the three other adult HT programmes (figure 1). The national paediatric HT programme at the RCH in Australia receives all paediatric patients from around the country. Thus, patients who are followed-up in area 2-where three Australian adult HT centres are located-are usually referred to this national paediatric HT centre if they are $<18$ years of age. We compared the cumulative proportion of Fontan failure and transplantation in patients with Fontan circulation between these two catchment areas.

Fontan failure was defined as death, HT, Fontan takedown or conversion to an extracardiac Fontan, PLE, plastic bronchitis or New York Heart Association functional class III or IV at follow-up. Since decision-making regarding (1) Fontan conversion, (2) Fontan takedown and (3) HT may potentially be institution-specific, we performed a separate analysis after exclusion of these three events as end points of Fontan failure. Hospital mortality was defined as death prior to hospital discharge or within 30 days of surgery. Primary graft dysfunction was defined as evidence of ventricular dysfunction with haemodynamic instability requiring high-dose inotropic support and/or a mechanical assist device within $24 \mathrm{~h}$ after transplantation. Stroke was defined as a focal neurological deficit of central origin that persisted beyond $24 \mathrm{~h}$ which was associated with positive imaging findings. Renal impairment was identified by pretransplant and post-transplant serum creatinine of $>100 \mu \mathrm{mol} / \mathrm{L}$. Renal failure was defined as the need for dialysis or doubling of pretransplant serum creatinine levels.

\section{Statistical analysis}

Continuous variables were compared using the unpaired t test, the Wilcoxon rank sum test and one-way analysis of variance. Data normality was assessed using the Shapiro-Wilk test. The Bonferroni post hoc test was used for multiple pairwise comparisons. Categorical variables were compared using the Fisher's exact and $\chi^{2}$ tests.

The Kaplan-Meier method was used to estimate survival and was compared using the log-rank statistic. Multivariable Cox regression was used to determine if catchment area was associated with likelihood of Fontan failure and undergoing HT. The covariates entered into the multivariable Cox regression model were: age at Fontan procedure, type of Fontan (atriopulmonary (AP), lateral tunnel (LT), extracardiac conduit (ECC)), time period (1975-1989, 1990-1999, 2000-2009, 20102015), ventricular morphology (left ventricle (LV), right ventricle (RV) dominant), hypoplastic left heart syndrome, number of surgical palliations prior to Fontan completion and prior bidirectional cavopulmonary connection.

Due to the non-linear relationship of age at Fontan with late outcomes, the age variable was analysed as four groups: $<3$ years, 3-5 years, 5-7 years and $>7$ years, with 3-5 years used as the reference group as we have previously done. ${ }^{1}$ Statistical analyses were conducted using SPSS V.23.0 (Armonk, New York, IBM).

\section{RESULTS}

\section{Comparison of catchment areas}

Of the 1369 Fontan patients discharged alive, 53\% (721/1369) were followed-up in area 1 and 47\% (648/1369) were followed-up in area 2. Overall mean follow-up time post-Fontan was $11 \pm 8$ years (13 408 patient-years). The characteristics of the patients between groups are presented in table 1 .

Patients in area 1 had a longer mean length of follow-up. There was no follow-up information available in $4.8 \%$ (67/ 1369) of patients, and this proportion was similar between areas (area 1: $4.3 \%, 31 / 721$ vs area $2: 5.6 \%, 36 / 648, \mathrm{p}=0.32$ ). Follow-up within the preceding 3 years was not available in $17 \%(237 / 1369)$ patients, and this was more often in those from area 2 (area 1: 14\%, 103/721 vs area 2: $21 \%, 134 / 648$, $\mathrm{p}=0.002$ ).

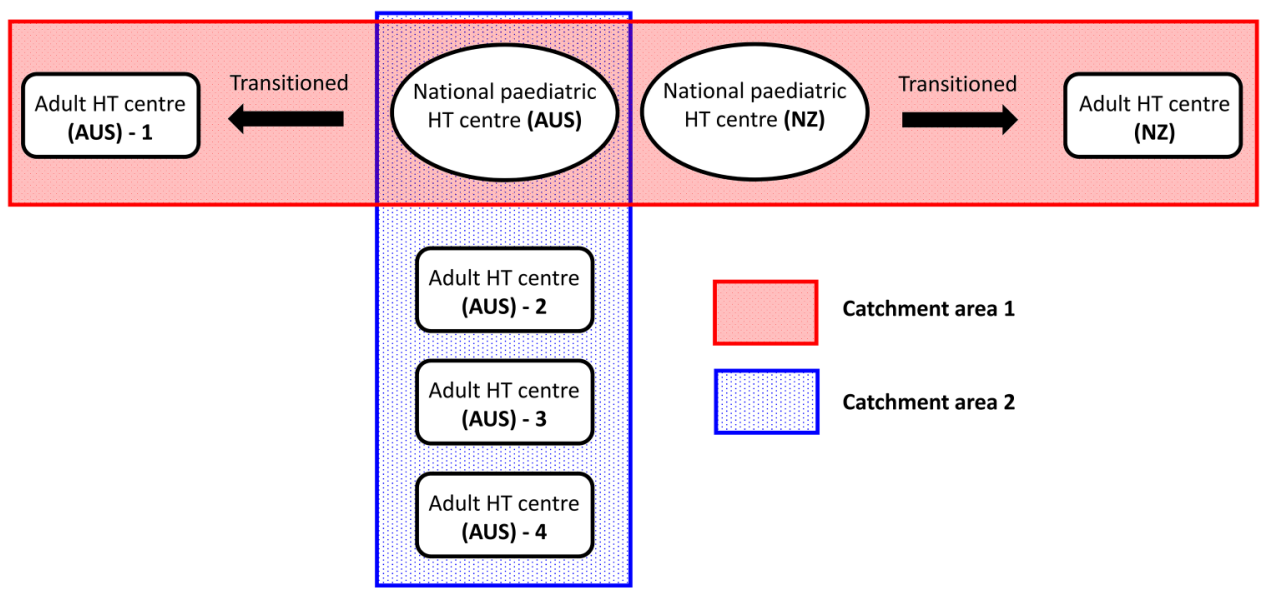

Figure 1 Graphical depiction of catchment areas: in area 1, patients are referred to either a national paediatric heart transplantation (HT) programme or its associated adult programme. In area 2, patients are referred to either a national paediatric HT programme or the three other adult HT programmes. The overlap exists as the national paediatric HT programme in Australia receives all paediatric patients referred from around the country. Thus, patients who are followed-up in area 2-where three Australian adult HT centres are located-are usually referred to this national paediatric HT centre if they are $<18$ years of age. AUS, Australia; NZ, New Zealand. 
Table 1 Characteristics at the time of Fontan procedure compared between areas of follow-up

\begin{tabular}{lccc}
\hline & $\begin{array}{l}\text { Area 1 } \\
(\mathbf{n}=721)\end{array}$ & $\begin{array}{c}\text { Area 2 } \\
(\mathbf{n}=648)\end{array}$ & $\mathbf{p}$ Value \\
\hline Male & $425(59)$ & $359(55)$ & 0.19 \\
Median age at Fontan in years & $4.6(3.5-6.2)$ & $4.8(3.8-6.4)$ & 0.034 \\
Median (IQR)* & & & \\
Fontan type & $124(17)$ & $81(13)$ & 0.015 \\
$\quad$ AP & $132(18)$ & $146(23)$ & 0.06 \\
LT & $458(64)$ & $415(64)$ & 0.87 \\
ECC & $7(1)$ & $6(1)$ & $>0.99$ \\
Unknown & & & \\
Time period & $112(16)$ & $74(11)$ & 0.028 \\
$\quad$ 1975-1989 & $183(25)$ & $155(24)$ & 0.57 \\
1990-1999 & $264(37)$ & $247(38)$ & 0.58 \\
2000-2009 & $162(22)$ & $172(27)$ & 0.089 \\
2010-2015 & & & \\
Morphology & $405(56)$ & $347(54)$ & 0.36 \\
$\quad$ LV & $216(30)$ & $189(29)$ & 0.77 \\
RV & $100(14)$ & $112(17)$ & 0.086 \\
$\quad$ Biventricular/indeterminate & $80(11)$ & $81(13)$ & 0.45 \\
HLHS & $2.0(1.1)$ & $1.8(1.0)$ & 0.001 \\
Palliations prior to Fontan-mean \\
(SD)
\end{tabular}

*Compared using non-parametric statistical testing.

tCompared using parametric statistical testing.

$\mathrm{AP}$, atriopulmonary; $\mathrm{BCPC}$, bi-directional cavo-pulmonary connection;

ECC, extracardiac conduit; HLHS, hypoplastic left heart syndrome; IQR, interquartile range; $L T$, lateral tunnel; LV, left ventricle; $R V$, right ventricle.

Overall freedom from documented Fontan failure was $89 \%$ $\pm 2.2 \%$ at 10 years and $74 \% \pm 3.9 \%$ at 20 years. The initial mode of failure was death in 62 patients, HT in 19 patients, Fontan takedown or conversion in 39 patients, PLE or plastic bronchitis in 40 patients and New York Heart Association functional class III or IV in 41 patients. Freedom from Fontan failure was higher in area 2 compared with area 1 as displayed in figure 2A. Patients from area 1 were more likely to have documented Fontan failure on multivariable Cox regression (HR 1.4, 95\% confidence interval (CI) 1.0 to $1.9, \mathrm{p}=0.035$ ).

Since decision-making regarding (1) Fontan conversion, (2) Fontan takedown and (3) HT may potentially be institutionspecific, we performed a separate analysis after exclusion of these three events as end points of Fontan failure. After exclusion of these events, overall freedom from Fontan failure was $91 \% \pm 2.0 \%$ at 10 years and $80 \% \pm 3.5 \%$ at 20 years. The initial mode of failure was death in 72 patients, PLE or plastic bronchitis in 41 patients and New York Heart Association functional class III or IV in 45 patients. This was similar between groups as displayed in figure $2 \mathrm{~B}$. There was no longer an association on multivariable analysis (HR 1.1, 0.8 to $1.6, \mathrm{p}=0.53$ ).

Using both the Australia and New Zealand Fontan Registry and the ANZCOTR, we identified 34 patients with a Fontan circulation who underwent HT. As displayed in figure 3, patients being followed-up in area 1 were more likely to receive HT compared with those in area 2 (area 1: 29/731 vs area 2: 5/648) with a cumulative proportion of $3.4 \% \pm 1.8 \%$ vs $0.7 \% \pm 0.8 \%$ at
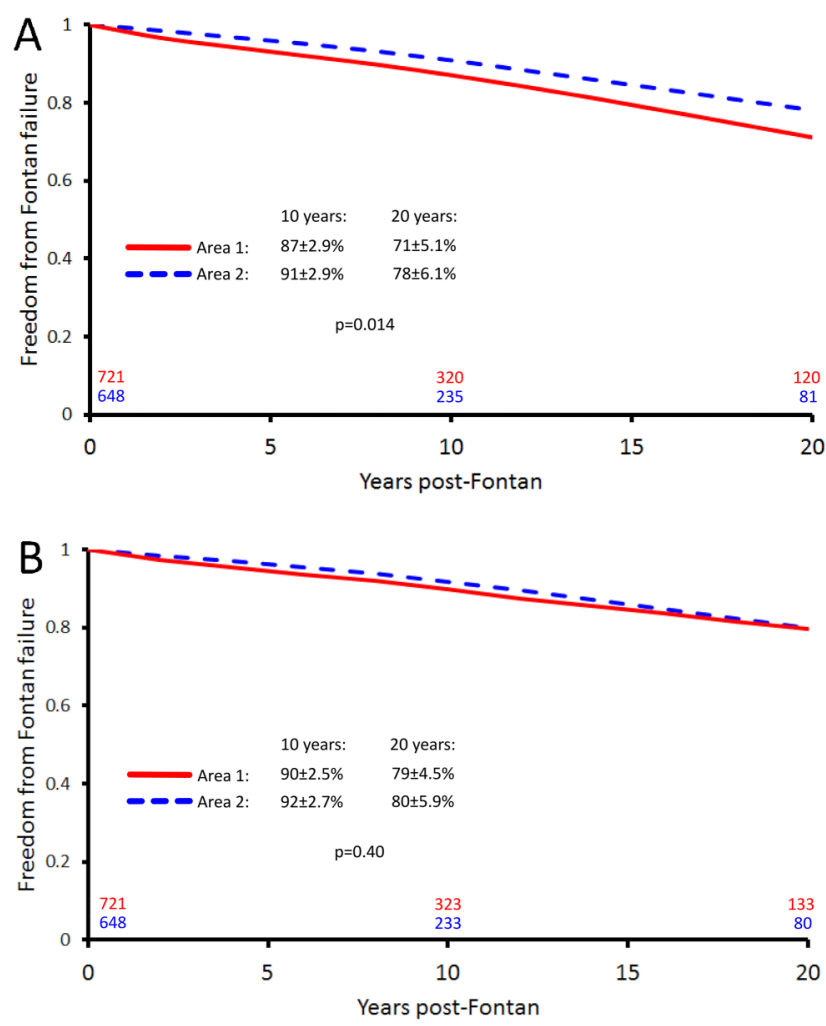

Figure 2 (A) Freedom from documented failure of Fontan circulation; (B) freedom from documented failure of Fontan circulation after excluding Fontan conversion, takedown and heart transplantation.

10 years and $6.8 \% \pm 3.0 \%$ vs $1.2 \% \pm 1.4 \%$ at 20 years $(\mathrm{p}=0.002)$. Multivariable Cox regression analysis of the 1369 Fontan patients demonstrated that patients living in area 1 were more likely to undergo HT (HR 4.7, 95\% CI 1.7 to 13.5 , $\mathrm{p}=0.003)$.

Comparison of patients followed-up in Australia $(n=1080)$ versus New Zealand $(n=289)$ showed similar rates of freedom from Fontan failure (Australia: $75 \% \pm 4.3 \%$ vs New Zealand: $69 \% \pm 9.4 \%$ at 20 years, $\mathrm{p}=0.45)$ and Fontan failure after exclusion of conversion, takedown and transplantation $(80 \% \pm 4.1 \%$ vs $79 \% \pm 7.6 \%$ at 20 years, $\mathrm{p}=0.86$ ). The cumulative rates of transplantation at 20 years were also similar between the two countries (Australia: 3.2\% $1.6 \%$ vs New Zealand: $2.3 \%$ $\pm 2.2 \%, \mathrm{p}=0.68$ ). There were no associations between country with Fontan failure or transplantation on multivariable analyses.

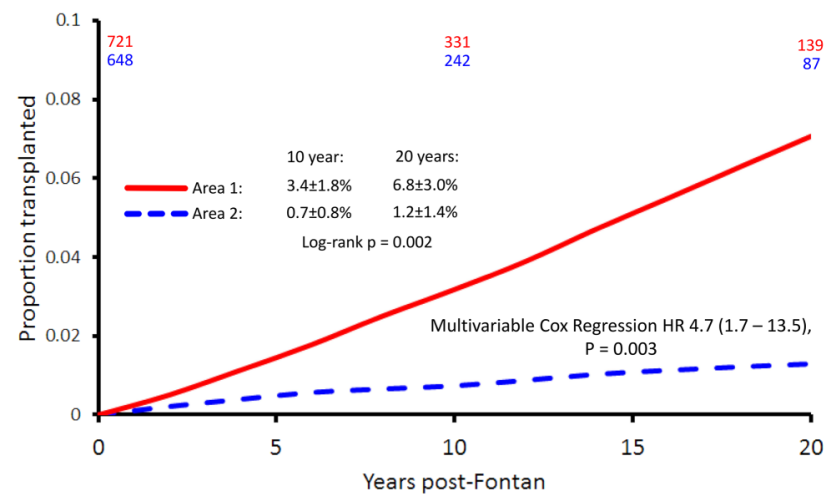

Figure 3 Cumulative proportion of heart transplantation in two catchment areas for 1369 patients with Fontan circulation in Australia and New Zealand. (Generated using the Kaplan-Meier method.) 


\section{Patients with a Fontan circulation undergoing HT}

All 34 heart transplants in patients with a Fontan circulation were performed at four out of a total of six transplant centres in Australia and New Zealand. Their characteristics are displayed in table 2. The age distribution was as follows: $0-6$ years $(n=3)$, $6-12$ years $(n=5), 12-18$ years $(n=10)$ and $>18$ years $(n=16)$. The indications for HT were chronic heart failure $(79 \%, 27 / 34)$, intractable supraventricular arrhythmia $(12 \%, 4 / 34)$ and PLE $(9 \%, 3 / 34)$.

Two patients were bridged to transplantation with a ventricular assist device. A Berlin Heart EXCOR device was used in a 5 -year old with hypoplastic left heart syndrome. A Thoratec paracorporeal device was used in a 26-year old patient with repaired double-inlet LV. The mean number of prior cardiac procedures was $2.7 \pm 1.1$ (median 3, range 1-5) and $62 \%$ (21/34) of patients had three or more prior cardiac procedures. All five patients with hypoplastic left heart syndrome had undergone the Norwood procedure followed by a bidirectional cavopulmonary shunt and Fontan completion.

Table 2 Characteristics of patients with a Fontan circulation $(n=34)$ undergoing heart transplantation

\begin{tabular}{|c|c|}
\hline & Fontan patients $(n=34)$ \\
\hline Age - median years (IQR) & 17 (11-31), range 4-51 \\
\hline \multicolumn{2}{|l|}{ Blood group } \\
\hline 0 & $17(50)$ \\
\hline$A$ & $10(29)$ \\
\hline B & $4(12)$ \\
\hline$A B$ & $3(9)$ \\
\hline \multicolumn{2}{|l|}{ Ventricular morphology } \\
\hline LV dominant & $19(56)$ \\
\hline RV dominant & $15(44)$ \\
\hline \multicolumn{2}{|l|}{ Diagnoses } \\
\hline Atrioventricular septal defect & $3(9)$ \\
\hline Double-inlet LV & $6(18)$ \\
\hline Double-outlet RV & $8(24)$ \\
\hline Hypoplastic left heart syndrome & $5(15)$ \\
\hline Transposition of the great arteries & $5(15)$ \\
\hline Tricuspid atresia & $7(21)$ \\
\hline Dextrocardia & $4(12)$ \\
\hline Situs inversus & $1(3)$ \\
\hline Left atrial isomerism & $2(6)$ \\
\hline Right atrial isomerism & $1(3)$ \\
\hline Time since Fontan (years) - mean (SD) & $11.5(9.1)$, range: $0.23-33$ \\
\hline \multicolumn{2}{|l|}{ Type of Fontan } \\
\hline ECC & $16(47)$ \\
\hline $\mathrm{AP}$ & $14(41)$ \\
\hline LT & $4(12)$ \\
\hline Fenestration & $10(29)$ \\
\hline Fenestration closure prior to $\mathrm{Tx}$ & $3(9)$ \\
\hline Number of prior surgeries-mean (SD) & $2.7(1.1)$ \\
\hline Bidirectional cavopulmonary connection & $13(38)$ \\
\hline Blalock-Taussig shunt & $5(15)$ \\
\hline Norwood procedure & $5(15)$ \\
\hline PLE & $5(15)$ \\
\hline Plastic bronchitis & $1(3)$ \\
\hline Pulmonary AVM & $1(3)$ \\
\hline History of atrial arrhythmia & $8(24)$ \\
\hline
\end{tabular}

$A P$, atriopulmonary Fontan; AVM, arteriovenous malformation; ECC, extracardiac conduit; IQR, interquartile range; LT, lateral tunnel Fontan; LV, left ventricle; PLE, protein-losing enteropathy; RV, right ventricle; $T x$, transplantation.
Operative details are presented in table 3 . Reconstruction of the aortic arch was required in 9\% (3/34) patients while pulmonary artery reconstruction was performed in 53\% (18/34). The mean cardiopulmonary bypass time was $282 \pm 99$ min while the mean donor heart ischaemic time was $291 \pm 91 \mathrm{~min}$.

Operative mortality was $5.8 \%(2 / 34)$. Two patients died due to sepsis (at 5 and 38 days postoperative). One patient who died in hospital was supported on extracorporeal membrane oxygenation (ECMO) for septic shock despite intact ventricular function. Primary graft failure occurred in $12 \%(4 / 34)$ patients, all of whom were supported with ECMO and recovered. Post-transplant renal impairment occurred in 29\% (10/34). Of these, seven required haemofiltration or haemodialysis, one patient required peritoneal dialysis and two were managed conservatively. No patient required permanent dialysis.

Mean follow-up was 7.0 \pm 6.0 years (median 4.7, range 0.1 21 years). Follow-up was $100 \%$ complete. There were six late mortalities. One patient was readmitted 38 days post-transplant and died of sepsis. Other patients died of cardiac allograft vasculopathy $(n=2)$, renal failure $(n=1), \operatorname{PLE}(n=1)$, which recurred 2 years after transplantation in a patient with preoperative PLE. The cause of death is unknown in one patient. Aside from the aforementioned patient who died of PLE, the other four patients had complete resolution of PLE. Kaplan-Meier survival is demonstrated in figure 4. Post-transplant survival at 1,5 and 10 years was $91 \%, 78 \%$ and $71 \%$, respectively.

\section{Comparison with patients with other forms of CHD identified by the ANZCOTR}

Of the 2479 heart transplants recorded in the ANZCOTR across six transplant centres, 139 had a diagnosis of CHD. Of those 139 patients, we identified 34 patients with a Fontan circulation and 87 patients without a Fontan circulation (27 univentricular, 60 biventricular) who underwent $\mathrm{HT}$ at the same four centres. The characteristics and outcomes of these patients are presented in table 3. A greater proportion of HT for Fontan patients occurred after January 2000 compared with those transplanted before this point $(36 \%(25 / 70)$ vs $18 \%$ (9/51), $\mathrm{p}=0.04)$. Fontan patients had longer cardiopulmonary bypass and donor ischaemic times. Survival analysis between Fontan patients and those with other CHD is presented in figure 4. Long-term survival after HT in patients with Fontan circulation was similar to survival after HT in other patients with CHD.

\section{DISCUSSION}

The number of patients living with a Fontan circulation is expected to grow. ${ }^{1}$ Data from the Australian and New Zealand Fontan Registry demonstrated survival of $76 \%$ at 25 years for the AP, $90 \%$ at 20 years for LT and $97 \%$ at 13 years for ECC Fontan. ${ }^{1}$ Nevertheless, the Fontan circulation will eventually fail in a significant number of patients. In more recent years, a greater proportion of patients with CHD referred for HT have a Fontan circulation. ${ }^{9}$ Similarly, in the current study the proportion of patients with a Fontan circulation was greater after 2000.

\section{Differences in rates of HT for patients with a Fontan circulation}

While previous studies have investigated factors associated with mortality for Fontan patients on transplant waiting lists, ${ }^{10}$ no study has specifically examined the differences in transplantation rates in the Fontan population at a national level. Patients with a failing Fontan circulation considered for HT often present with co-morbidities that place them at higher risk of postoperative morbidity and mortality such as hepatic and renal dysfunction, PLE 
Table 3 Perioperative characteristics and early outcomes in patients with Fontan circulation compared with those with other forms of congenital heart disease

\begin{tabular}{|c|c|c|c|c|c|c|c|}
\hline Patients' details & Fontan $(n=34)$ & Other univentricular $(n=27)$ & * & Biventricular $(n=60)$ & $t$ & $\ddagger$ & p Value \\
\hline Age—-median years, (IQR)§ & $17(11-31)$ & $14(5-24)$ & 0.065 & $20(14-41)$ & 0.26 & 0.011 & 0.042 \\
\hline Weight median kg (IQR)§ & $58(26-73)$ & $32(15-65)$ & 0.054 & $60(38-74)$ & 0.45 & 0.005 & 0.007 \\
\hline \multicolumn{8}{|l|}{ Univentricular } \\
\hline LV dominant & $19(56)$ & $14(52)$ & $>0.99$ & - & - & - & $>0.99$ \\
\hline RV dominant & $15(44)$ & $13(48)$ & $>0.99$ & - & - & - & $>0.99$ \\
\hline Hypoplastic left heart syndrome & $6(18)$ & $7(26)$ & 0.53 & - & - & - & 0.53 \\
\hline \multicolumn{8}{|l|}{ Biventricular } \\
\hline TGA & - & - & - & $14(23)$ & - & - & - \\
\hline ccTGA & - & - & - & $10(17)$ & - & - & - \\
\hline TOF & - & - & - & 7 (12) & - & - & - \\
\hline Ebstein's anomaly & - & - & - & $4(7)$ & - & - & - \\
\hline Aortic stenosis & - & - & - & $4(7)$ & - & - & - \\
\hline Mitral regurgitation & - & - & - & $3(5)$ & - & - & - \\
\hline AVSD & - & - & - & $4(7)$ & - & - & - \\
\hline VSD & - & - & - & $9(15)$ & - & - & - \\
\hline Othersף & - & - & - & $5(8)$ & - & - & - \\
\hline Days on waiting list-median (IQR)§ & $179(112-499)$ & $130(72-290)$ & 0.24 & $133(62-307)$ & 0.087 & 0.79 & 0.89 \\
\hline Previous cardiac surgery & $34(100)$ & $24(89)$ & 0.081 & $52(87)$ & 0.047 & $>0.99$ & 0.089 \\
\hline Number of prior surgeries-mean $(S D)^{* *}$ & $2.7(1.1)$ & $2.4(1.7)$ & $>0.99$ & $1.9(1.2)$ & 0.006 & 0.18 & 0.006 \\
\hline Preoperative inotropes & $4(12)$ & $3(11)$ & $>0.99$ & $7(12)$ & $>0.99$ & $>0.99$ & $>0.99$ \\
\hline Preoperative VAD & $2(6)$ & $0(0)$ & 0.13 & $8(13)$ & 0.32 & 0.054 & 0.094 \\
\hline Preoperative creatinine ( $\mu \mathrm{mol} / \mathrm{L})$ - mean $(\mathrm{SD})^{* *}$ & $78(42)$ & $56(27)$ & 0.11 & $79(51)$ & $>0.99$ & 0.043 & 0.038 \\
\hline \multicolumn{8}{|l|}{ Operative } \\
\hline Cardiopulmonary bypass time-mean $\min (\mathrm{SD})^{* *}$ & $282(99)$ & $209(82)$ & 0.012 & $207(93)$ & 0.001 & $>0.99$ & 0.001 \\
\hline Ischaemic time-mean $\min (S D)^{* *}$ & $291(91)$ & $230(92)$ & 0.064 & $233(100)$ & 0.022 & $>0.99$ & 0.016 \\
\hline Great artery reconstruction & $19(56)$ & $10(37)$ & 0.20 & $0(0)$ & $<0.001$ & $<0.001$ & $<0.001$ \\
\hline Aortic arch reconstruction & $3(9)$ & $3(11)$ & $>0.99$ & $0(0)$ & 0.045 & 0.035 & 0.041 \\
\hline Pulmonary artery repair/reconstruction & $18(53)$ & $7(26)$ & 0.04 & $0(0)$ & $<0.001$ & $<0.001$ & $<0.0001$ \\
\hline \multicolumn{8}{|l|}{ Early outcomes } \\
\hline In-hospital mortality & $2(6)$ & $4(15)$ & 0.39 & $5(8)$ & $>0.99$ & 0.45 & 0.46 \\
\hline Primary graft failure & $4(12)$ & $4(15)$ & $>0.99$ & $10(17)$ & 0.76 & $>0.99$ & 0.81 \\
\hline Stroke & $1(3)$ & $1(4)$ & $>0.99$ & $3(5)$ & $>0.99$ & $>0.99$ & 0.88 \\
\hline Renal impairment & $11(32)$ & $5(19)$ & 0.25 & $14(23)$ & 0.35 & 0.78 & 0.43 \\
\hline Post-transplant ECMO & $5(15) \dagger \dagger$ & $3(11)$ & $>0.99$ & $8(13)$ & $>0.99$ & $>0.99$ & 0.92 \\
\hline Post-transplant IABP & $3(9)$ & $2(7)$ & $>0.99$ & $7(12)$ & 0.74 & 0.71 & 0.80 \\
\hline
\end{tabular}

Categorical variables displayed as number (percentage).

${ }^{*}$ Comparison between Fontan and other univentricular repairs.

tComparison between Fontan and biventricular repairs. $¥$ Comparison between other univentricular and biventricular repairs.

$\S$ Compared using non-parametric statistical testing.

${ }^{* *}$ Compared using parametric statistical testing.

10thers: these five patients had: truncus arteriosus, tricuspid dysplasia, VSD with partial Uhl's anomaly, partial anomalous pulmonary venous drainage, anomalous left main coronary artery.

t†One patient was placed on ECMO due to septic shock despite intact graft function.

AVSD, atrioventricular septal defect; ECMO, extracorporeal membrane oxygenation; IABP, intra-aortic balloon pump; IQR, interquartile range; LV: left ventricle; RV: right ventricle; TGA,

transposition of the great arteries; ccTGA, congenitally corrected TGA; TOF, tetralogy of Fallot; VAD, ventricular assist device; VSD, ventricular septal defect;.

and plastic bronchitis. ${ }^{15}$ Reoperative cardiac surgery is inherently risky. Difficult intraoperative dissection of distorted thoracic structures and reconstruction of the great vessels at the time of HT are often required. This results in longer cardiopulmonary bypass and donor ischaemic times which contribute towards perioperative morbidity. ${ }^{5} 11$ Demanding perioperative management, coupled with early reports of greater mortality after HT meant that patients with Fontan circulation may have been perceived to be of higher perioperative risk by transplantation programmes. All these may have limited the general access of patients with a Fontan circulation to HT, particularly in adult centres where recipient numbers are larger than the potential donor pool.

To our knowledge, this study is the first to investigate the rates of transplantation for patients with a Fontan circulation at a national level. It appears from our study that patients living in areas which refer to either a national paediatric HT programme or its associated adult programme (area 1 ) are more likely to receive HT.

Despite regional variations in the rate of HT in the Fontan population, the overall rate of HT is low in all areas. Even in area 1, freedom from Fontan failure was $71 \%$ at 20 years, yet the cumulative proportion transplanted at 20 years was only $6.8 \%$. While not all patients with Fontan failure are suitable for HT, a substantial portion of patients with Fontan failure nonetheless did not undergo HT.

Patients from area 1 were statistically more likely to have Fontan failure compared with those in area 2. However, this was due to the fact that more Fontan takedown, conversion and HT procedures were performed in area 1. After exclusion of these procedures there was no association between the area and likelihood of Fontan failure. 


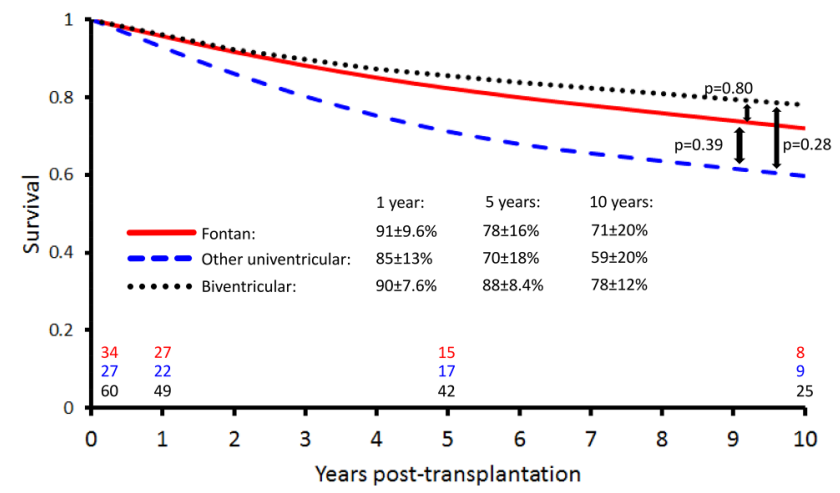

Figure 4 Survival after heart transplantation in Fontan patients compared and patients with other forms of congenital heart disease.

\section{Results of HT in patients with a Fontan circulation}

In a multicentre study of 488 who underwent HT from 1990 to 2002, Lamour et al ${ }^{3}$ compared 107 patients with a Fontan circulation undergoing cardiac transplantation with 381 patients with other forms of CHD and found that patients with a Fontan circulation were 8.6 times more likely to die after HT. Five-year survival was $60 \%$ in the Fontan group compared with $74 \%$ in the other CHD group. Other groups have also reported similar results. ${ }^{9}{ }^{12}$ These findings initially raised concerns regarding $\mathrm{HT}$ in these patients.

Recently, individual centres have reported more favourable early results (operative mortality of $\leq 15 \%$ ) in HT for patients with a failing Fontan. ${ }^{5} 1314$ The relatively low operative mortality $(6 \%)$ seen in our study may be related to several factors. There was a small number of patients with PLE (15\%) compared with higher proportions of up to $50 \%$ in other studies. ${ }^{5} 1215$ Only one of the patients in our series underwent transplantation within the first year for early Fontan failure. Of the 1369 patients in the Fontan Registry, the 34 who underwent HT may represent a selected, robust group who were deemed more appropriate for HT. We did not have detailed information on the number of patients who were diagnosed with Fontan failure and were managed conservatively or palliated.

Kovach et al, ${ }^{2}$ using the Paediatric Heart Transplant Study database, analysed HT performed for CHD at 32 institutions from 1992 to 2008. Patients with a Fontan circulation $(n=194)$ were compared with those who had undergone a Glenn shunt $(n=189)$ and those with other forms of CHD $(n=421)$. They found similar long-term survival after HT between the groups. This was consistent with our study. While these results are encouraging, differences in survival after HT may nevertheless reflect selection bias. Patients with a Fontan circulation who are the most critically ill may not have been referred for or survived to HT.

\section{Strategies in HT for patients with Fontan circulation}

Given the foreseeable growth in the number of patients with a Fontan circulation referred for $\mathrm{HT}^{9}{ }^{9}$ we believe that transplant teams should adjust their practice to deal with these complex patients. We typically transfer patients with a Fontan circulation to the operating room at least $4 \mathrm{~h}$ prior to the donor heart's estimated arrival. This time is usually necessary to perform extensive dissection and reconstruction of the great vessels. ${ }^{11}$ The coordination with the heart retrieval team must be precise to minimise unnecessary donor ischaemic time. Surgeons who perform HT in patients with CHD must have a thorough understanding of the relevant anatomy, in particular the distorted intrathoracic structures that exist following multiple univentricular palliation procedures.

\section{LIMITATIONS}

This study is limited by its retrospective nature. This study involved a long study period, with perioperative strategies changing over time. Furthermore, we may not have complete information on the patients who were diagnosed with Fontan failure and were palliated without referral to HT.

\section{CONCLUSION}

Although HT after the Fontan procedure can be achieved with excellent outcomes, most patients with Fontan failure do not undergo HT. Significant regional differences in rates of HT in Fontan patients exist.

\section{Key messages}

\section{What is already known on this subject?}

A proportion of patients living with a Fontan circulation will require heart transplantation (HT). Such patients are often perceived to be of higher operative risk compared with other potential transplant candidates with congenital heart disease (CHD).

\section{What might this study add?}

This study is the first to investigate the rates and outcomes of transplantation for patients with a Fontan circulation at a national level. Patients living in areas which refer to either a national paediatric $\mathrm{HT}$ programme or its affiliated adult programme are more likely to undergo HT. The overall rate of $\mathrm{HT}$ for Fontan patients is low in all areas. A substantial portion of patients with Fontan failure do not undergo HT.

\section{How might this impact on clinical practice?} Our study suggests that a centralised programme with unified indications for $\mathrm{HT}$ and expertise in surgical management of heart failure in CHD may result in greater access to transplantation for patients with failing Fontan circulation of all ages.

Acknowledgements This work was partially supported by a National Health and Medical Research Council Partnership grant (app 1076849). WS is supported by the Royal Australasian College of Surgeons Foundation for Surgery Peter King/Heart Foundation Research Scholarship in addition to the University of Melbourne Viola Edith Reid and the RG and AU Meade Scholarships. Yd'U is a Clinician Practitioner Fellow of the National Health and Medical Research Council of Australia (1082186). There are no relationships with industry.

Contributors All authors have contributed significantly to the manuscript. All authors agree to be accountable for all aspects of the work in ensuring that questions related to the accuracy or integrity of any part of the work are appropriately investigated and resolved. The contribution of each author is substantiated below: WYS was responsible for conception and design of the study, data collection, interpretation of data, drafting and revision of the manuscript for important intellectual content and final approval of the manuscript. MSY was responsible for conception and design of the study, data collection, interpretation of data, drafting and revision of the manuscript for important intellectual content and final approval of the manuscript. DCMG was responsible for conception and design of the study, data collection, interpretation of data, revision of the manuscript for important intellectual content and final approval of the manuscript. PJ was responsible for data collection, interpretation of data, revision of the manuscript for important intellectual content and final approval of the manuscript. PNR was responsible for conception and design of the study, data collection, interpretation of data, revision of the manuscript for important intellectual content and final approval 
of the manuscript. SFM was responsible for data collection, interpretation of data, revision of the manuscript for important intellectual content and final approval of the manuscript. KF was responsible for design of the study, data collection, interpretation of data, revision of the manuscript for important intellectual content and final approval of the manuscript. AK was responsible for data collection, interpretation of data, revision of the manuscript for important intellectual content and final approval of the manuscript. Y $Y$ ' $U$ was responsible for conception and design of the study, interpretation of data, drafting and revision of the manuscript for important intellectual content and final approval of the manuscript. RGW was responsible for conception and design of the study, data collection, interpretation of data, drafting and revision of the manuscript for important intellectual content and final approval of the manuscript. IEK was responsible for conception and design of the study, collection of data, interpretation of data, drafting and revision of the manuscript for important intellectual content and final approval of the manuscript.

Funding Department of Health, Australian Government. National Health and Medical Research Council (Clinician Practitioner Fellow-Yd'U 1082, Partnership Grant 1076849). National Heart Foundation of Australia (Health Professional Scholarship-WS). Royal Australasian College of Surgeons (Foundation for Surgery Peter King Scholarship-WS).

Competing interests None declared.

Ethics approval Hospital research and ethics committees of respective participating institutions, see http://www.fontanregistry.com/.

Provenance and peer review Not commissioned; externally peer reviewed.

\section{REFERENCES}

1 d'Udekem Y, lyengar AJ, Galati JC, et al. Redefining expectations of long-term survival after the Fontan procedure: twenty-five years of follow-up from the entire population of Australia and New Zealand. Circulation 2014;130(Suppl 1): S32-8.

2 Kovach JR, Naftel DC, Pearce FB, et al. Comparison of risk factors and outcomes for pediatric patients listed for heart transplantation after bidirectional Glenn and after Fontan: an analysis from the Pediatric Heart Transplant Study. J Heart Lung Transplant 2012;31:133-9.
3 Lamour JM, Kanter KR, Naftel DC, et al. The effect of age, diagnosis, and previous surgery in children and adults undergoing heart transplantation for congenital heart disease. J Am Coll Cardiol 2009;54:160-5.

4 Jayakumar KA, Addonizio LJ, Kichuk-Chrisant MR, et al. Cardiac transplantation after the Fontan or Glenn procedure. J Am Coll Cardiol 2004;44:2065-72.

5 Davies RR, Sorabella RA, Yang J, et al. Outcomes after transplantation for "failed" Fontan: a single-institution experience. I Thorac Cardiovasc Surg 2012;143:1183-92.e4.

6 lyengar AJ, Winlaw DS, Galati JC, et al. The Australia and New Zealand Fontan Registry: description and initial results from the first population-based Fontan registry. Intern Med J 2014;44:148-55.

7 lyengar AJ, Winlaw DS, Galati JC, et al. Trends in Fontan surgery and risk factors for early adverse outcomes after Fontan surgery: the Australia and New Zealand Fontan Registry experience. J Thorac Cardiovasc Surg 2014;148:566-75.

8 lyengar AJ, Winlaw DS, Galati JC, et al. The extracardiac conduit Fontan procedure in Australia and New Zealand: hypoplastic left heart syndrome predicts worse early and late outcomes. Eur J Cardiothorac Surg 2014;46:465-73; discussion 473.

9 Voeller RK, Epstein DJ, Guthrie TJ, et al. Trends in the indications and survival in pediatric heart transplants: a 24-year single-center experience in 307 patients. Ann Thorac Surg 2012;94:807-15; discussion 815-16.

10 Bernstein D, Naftel D, Chin C, et al. Outcome of listing for cardiac transplantation for failed Fontan: a multi-institutional study. Circulation 2006;114:273-80.

11 lyengar AJ, Sharma VJ, Weintraub RG, et al. Surgical strategies to facilitate heart transplantation in children after failed univentricular palliations: the role of advanced intraoperative surgical preparation. Eur I Cardiothorac Surg 2014;46:480-5.

12 Backer $C L$, Russell HM, Pahl E, et al. Heart transplantation for the failing Fontan. Ann Thorac Surg 2013;96:1413-19.

13 Kanter KR, Mahle WT, Vincent RN, et al. Heart transplantation in children with a Fontan procedure. Ann Thorac Surg 2011;91:823-9.

14 Griffiths ER, Kaza AK, Wyler von Ballmoos MC, et al. Evaluating failing Fontans for heart transplantation: predictors of death. Ann Thorac Surg 2009;88:558-63.

15 Michielon G, van Melle JP, Wolff D, et al. Favourable mid-term outcome after heart transplantation for late Fontan failure. Eur J Cardiothorac Surg 2015;47: 665-71. 